\title{
On the Low-Temperature Diffusion of Localized Frenkel Excitons in Linear Molecular Aggregates
}

\author{
A. V. Malyshev, \\ GISC, Departamento de Física de Materiales, Universidad Complutense, E-28040 Madrid, Spain
}

Received: January 16, 2003; In Final Form: March 10, 2003

\begin{abstract}
We study theoretically the diffusion of one-dimensional Frenkel excitons in J-aggregates at temperatures that are smaller or on the order of the J-bandwidth. We consider an aggregate as an open linear chain with uncorrelated on-site (diagonal) disorder that localizes the exciton at chain segments of size smaller than the full chain length. The exciton diffusion over the localization segments is considered as incoherent hopping. The diffusion is probed by the exciton fluorescence quenching, which is due to the presence of point traps in the aggregate. The rate equation for populations of the localized exciton states is used to describe the exciton diffusion and trapping. We show that there exist two regimes of the exciton diffusion at low temperatures. The first, slower one, involves only the states of the very tail of the density of states, while the second, much faster one, also involves the higher states that are close to the bottom of the bare exciton band. The activation energy for the first regime of diffusion is on the order of $1 / 4$ of the J-bandwidth, while for the second one, it is on the order of the full J-bandwidth. We discuss also the experimental data on the fast low-temperature exciton-exciton annihilation reported recently by I. G. Scheblykin et al. J. Phys. Chem. B 2000, 104, 10949.
\end{abstract}

\section{Introduction}

Since the seminal works by Jelley ${ }^{1}$ and Scheibe, ${ }^{2}$ the concept of Frenkel excitons ${ }^{3-5}$ has been used for explaining the remarkable optical properties of molecular J-aggregates: (i) the appearance of a narrow and intense line in the red wing of the absorption spectra (so-called J-band), the full width of which is on the order of several tens of wavenumbers at cryogenic temperatures and (ii) the increase of the oscillator strength of the optical transition by almost 2 orders of magnitude. ${ }^{6-10}$ During the 1990s, a considerable progress in understanding of linear and nonlinear optical dynamics of J-aggregates was made (for details, see the reviews in refs 11-13 and references therein). Although monomers that form the aggregates have complex chemical structure, both linear and nonlinear optical dynamics in J-aggregates have been successfully described on the basis of the simplest one-dimensional (1D) tight-binding model with diagonal or off-diagonal disorder or both, both uncorrelated $^{10,14,15}$ and correlated. ${ }^{14,16-18}$

The eigenstates of a homogeneous (nondisordered) $\mathrm{J}$-aggregate extend over the whole ( $N$ monomers) aggregate. Disorder localizes the lowest in energy exciton states at segments of about $N^{*}$ molecules; $N^{*}$ depends on the disorder magnitude and is typically much smaller than the total number of molecules in the chain: $N^{*} \ll N$. One of the most important consequences of this localization is the appearance of states below the bottom of the bare exciton band. These states form the tail of the density of states (DOS) and carry almost the whole oscillator strength of the aggregate. For this reason, the one-exciton absorption in $\mathrm{J}$-aggregates is spectrally located at the tail of the DOS (see, for instance, refs 9 and 10) and the width of the absorption band is on the order of the width of the DOS tail.

\footnotetext{
* To whom correspondence should be addressed.

$\doteqdot$ On leave from Ioffe Physiko-Technical Institute, 26 Politechnicheskaya str., 194021 Saint-Petersburg, Russia.

On leave from S. I. Vavilov State Optical Institute, Saint-Petersburg,
} Russia.
The exciton diffusion in a disordered aggregate is essentially the transition from one localized eigenstate to another. The transition probability depends particularly on the temperature, the energy spacing between the involved states, and the overlap of these states. The lower states, being localized at different $N^{*}$ molecule segments of the aggregate, overlap very weakly. ${ }^{19}$ Contrary to that, the higher exciton states, which are localized at segments larger than $N^{*}$ molecules, overlap strongly with several lower tail states. Although higher states are thermally less favorable, the hops from the lower to higher states can be faster than those between the lower states because of the higher overlap. In this paper, we show that this competition between the overlaps and thermal favorability results in a complex scenario of the exciton transport at low temperatures. At zero temperature, an exciton resides in one of the lower states at the tail of the DOS. As temperature rises, first, the exciton starts to diffuse over the weakly overlapped states of the DOS tail. The activation energy for this regime is on the order of $1 / 4$ of the DOS tail width. The diffusion in this regime is very slow. As the temperature increases further, the higher states come into play. As these states overlap much better with the lower states and each other and also are more extended, the diffusion rate increases by several orders of magnitude. The activation energy for this faster regime of the exciton diffusion is on the order of the DOS tail width or, in other words, on the order of the J-bandwidth.

To the best of our knowledge, these aspects of the 1D diffusion problem have not been discussed in the literature yet. The same tight-binding Hamiltonian was used to describe transport properties of electrons in doped semiconductors ${ }^{20}$ as well as those of optical excitations in activated glasses. ${ }^{21,22}$ It is to be stressed that, despite the seeming similarity of these problems, the outlined scenario of the low-temperature 1D diffusion over the localization segments is more complex than the diffusion over one-level point impurity centers in semiconductors or glasses. The major complication comes from the fact 
that the exciton can hop sideways to a different segment not only directly (a "horizontal" hop) but also indirectly via higher states, so "vertical" hops up in energy become extremely important. In fact, it is the indirect hops that provide the dominant contribution to the diffusion rate at temperatures on the order of the J-bandwidth.

We use the quenching of the exciton fluorescence by point traps to probe the exciton diffusion. The temperature range lower or on the order of magnitude of the J-bandwidth is of our primary interest; higher temperatures are beyond the scope of the present work.

The outline of the paper is as follows. In section 2, we present the microscopic model of exciton trapping. Section 3 is focused on the qualitative discussion of the channels of the exciton diffusion over the localization segments. The results of our numerical simulations of the exciton fluorescence quenching, obtained on the basis of the rate equation approach, are the contents of section 4 . In section 5 , we conclude the paper and discuss the results of the recent experiments on the fast excitonexciton annihilation in the aggregates of the triethylthiacarbocyanine salt of 3,3'-bis(sulfopropyl)-5,5'-dichloro-9-ethylthiacarbocyanine (THIATS). ${ }^{23}$

\section{Microscopic Model of the Exciton Fluorescence Quenching}

We model a J-aggregate by $N(N \gg 1)$ optically active twolevel molecules forming a regular in space $1 \mathrm{D}$ open chain. The corresponding Frenkel exciton Hamiltonian reads 4 (for the sake of simplicity only the nearest-neighbor interaction is considered)

$$
H=\sum_{n=1}^{N} E_{n}|n\rangle\langle n|-J \sum_{n=1}^{N-1}(|n+1\rangle\langle n|+| n\rangle\langle n+1|)
$$

Here, $E_{n}$ is the excitation energy of the $n$th molecule; $|n\rangle$ denotes the state vector of the $n$th excited molecule. The energies $E_{n}$ are assumed to be Gaussian uncorrelated (for different sites) stochastic variables distributed around the mean value $\omega_{0}$ (which is set to zero without loss of generality) with the standard deviation $\Delta$. The hopping integral, $-J$, is considered to be nonrandom and negative $(J>0)$, which corresponds to the case of J-aggregates (e.g., see ref 6). In this case, the states coupled to the light are those close to the bottom of the exciton band. In what follows, moderate disorder $(\Delta<J)$ is considered. This implies that the exciton eigenstates, $\varphi_{v}(v=1,2, \ldots, N)$, found from the Schrödinger equation

$$
\sum_{m=1}^{N} H_{n m} \varphi_{v m}=\epsilon_{v} \varphi_{v n}, \quad H_{n m}=\langle n|H| m\rangle
$$

are extended over relatively large segments of the chain. However, the typical size of these localization segments, $N^{*}$, is small compared to the full chain length $N$ (units of the lattice constant are used throughout the paper).

Having been excited into an eigenstate $v$, an exciton cannot hop to other eigenstates if coupling to vibrations is not taken into account. We assume that this coupling is weak and do not consider polaron effects. This limit is applicable to a number of J-aggregates because the Stokes shift of the luminescence spectra with respect to the absorption spectra is usually small. ${ }^{7,9}$ The exciton-vibration interaction causes the incoherent hopping of excitons from one eigenstate to another. Only one-phonon processes are considered throughout the paper. The hopping that results from the overlap of homogeneously broadened exciton levels is a two-phonon process, and therefore, it is beyond the scope of the present paper. We take the hopping rate from the state $v$ to the state $\mu$ in the following form (e.g., see ref 24)

$$
\begin{aligned}
& W_{\mu \nu}=W_{0} S\left(\left|\epsilon_{v}-\epsilon_{\mu}\right|\right) \sum_{n=1}^{N} \varphi_{\nu n}{ }^{2} \varphi_{n \mu}{ }^{2} \\
& \times \begin{cases}n\left(\epsilon_{\mu}-\epsilon_{v}\right) & \epsilon_{\mu}>\epsilon_{v} \\
1+n\left(\epsilon_{v}-\epsilon_{\mu}\right) & \epsilon_{\mu}<\epsilon_{v}\end{cases}
\end{aligned}
$$

Here, the constant $W_{0}$ characterizes the amplitude of hopping and $n(\epsilon)=[\exp (\epsilon / T)-1]^{-1}$ is the occupation number of the vibration mode with the energy $\epsilon$ (the Boltzmann constant is set to unity). Because of the presence of the $n(\epsilon)$ and $1+n(\epsilon)$ factors, the rate $W_{\mu \nu}$ meets the principle of detailed balance: $W_{\mu \nu}=W_{v \mu} \exp \left[\left(\epsilon_{v}-\epsilon_{\mu}\right) / T\right]$. Thus, in the absence of decay channels, the eventual exciton distribution is the Boltzmann equilibrium distribution. The sum over sites in eq 3 represents the overlap integral of exciton probabilities for the states $\mu$ and $\nu$. The spectral factor $S\left(\left|\epsilon_{v}-\epsilon_{\mu}\right|\right)$ depends on the details of the exciton-phonon coupling, as well as on the DOS of the medium into which the aggregate is embedded. For example, within the Debye model for the density of phonon states, this factor takes the form $S\left(E_{v}-E_{\mu}\right)=\left(\left|E_{v}-E_{\mu}\right| / J\right)^{3}{ }^{25}$ However, this model is applicable to glassy media (the media we assume as the host) only in a narrow frequency interval on the order of several wavenumbers (see, for instance, refs 26 and 27). Therefore, as in refs 28 and 29, we restrict ourselves to a linear approximation to this factor, $S\left(E_{v}-E_{\mu}\right)=\left|E_{v}-E_{\mu}\right| / J$. This accounts for reduction of the exciton-vibration interaction in the long-wave acoustic limit. ${ }^{4,5}$ Also, it eliminates the divergence of $W_{\nu \mu}$ at small values of $\left|E_{v}-E_{\mu}\right|$.

The diffusion of Frenkel excitons can be probed by quenching of the exciton fluorescence by traps. Consider an aggregate with point traps, namely monomers at which an exciton decays nonradiatively and very fast compared to the typical spontaneous emission rate of the aggregate. Then, those excitons that reach the traps decay nonradiatively and contribute to the fluorescence quenching. If an exciton is created far from the trap, it has to diffuse to the trap to be quenched; the faster it diffuses the more effective is the fluorescence quenching. Thus, the quenching rate depends on the diffusion rate and can be used as a probe of the latter.

We define the quenching rate of the exciton state $v$ as

$$
\Gamma_{v}=\Gamma \sum_{i=1}^{N_{\mathrm{q}}}\left|\varphi_{v i}\right|^{2}
$$

where $\Gamma$ is the amplitude of exciton quenching and the sum runs over positions of the $N_{\mathrm{q}}$ traps. Thus, we take the quenching rate to be proportional to the probability to find the exciton at trap sites. We assume that point traps do not change either the disorder configuration or the exciton eigenfunctions, $\varphi_{v i}$.

We describe the process of the exciton trapping by means of the rate equation:

$$
\dot{P}_{v}=-\left(\gamma_{v}+\Gamma_{v}\right) P_{v}+\sum_{\mu=1}^{N}\left(W_{\nu \mu} P_{\mu}-W_{\mu \nu} P_{v}\right)
$$

where $P_{v}$ is the population of the $v$ th exciton eigenstate and the dot denotes the time derivative, $\gamma_{v}=\gamma f_{v}$ is the spontaneous emission rate of the $v$ th exciton state, and $\gamma$ is that of a monomer, $f_{v}=\left(\sum_{n=1}^{N} \varphi_{v n}\right)^{2}$ being the oscillator strength of the state $v$. The initial total population is normalized to unity: $\sum_{\nu} P_{\nu}(0)=1$. 
The temperature dependence of the exciton quenching is calculated as follows. We admit the definition of the exciton fluorescence decay time as the integrated total population:

$$
\tau=\int_{0}^{\infty} \mathrm{d} t\left\langle\sum_{v=1}^{N} P_{v}(t)\right\rangle
$$

where angle brackets denote the average over disorder realizations and traps positions. The lifetime defined as eq 6 is the expectation value of the photon emission time and, therefore, is appropriate for nonexponential fluorescence kinetics, the case with which we are dealing (see ref 25 for detailed discussion).

The decay time has to be calculated for aggregates with traps (denote it as $\tau$ ) and without traps (denote it as $\tau_{0}$ ). The quenching rate is then defined as

$$
W_{\mathrm{q}}=\frac{1}{\tau}-\frac{1}{\tau_{0}}
$$

This quantity carries information about the diffusion rate and is the object of our analysis.

The definition of the decay rate as the integrated total population allows for considerable simplification of the calculation procedure. Write the solution of eq 5 in the formal matrix form

$$
P_{\nu}(t)=\sum_{\mu=1}^{N}\left(\mathrm{e}^{-\hat{R} t}\right)_{\nu \mu} P_{\mu}(0)
$$

where

$$
R_{v \mu}=\left(\gamma_{v}+\Gamma_{v}+\sum_{v^{\prime}=1}^{N} W_{v^{\prime} v}\right) \delta_{\mu \nu}-W_{v \mu}
$$

After substitution of eq 8 into eq 6 and integration over time, $\tau$ can be expressed in terms of the $\hat{R}$-matrix:

$$
\tau=\left\langle\sum_{\nu, \mu=1}^{N} \hat{R}_{v \mu}{ }^{-1} P_{\mu}(0)\right\rangle
$$

Calculation of the quenching rate $W_{\mathrm{q}}$ requires computation of the inverse matrix $\hat{R}^{-1}$ for each realization of disorder rather than the fluorescence kinetics. The inverse matrix is to be found twice, for an aggregate with and without traps. Note that the decay time $\tau_{0}$ also depends on temperature (see, for example, refs 25,29 , and 30).

\section{Qualitative Arguments}

At low temperatures, excitons reside in the tail of the DOS, that is, below the bottom of the bare exciton band, $E=-2 \mathrm{~J}$. As we show subsequently, higher states that are close to the bottom of the bare band contribute to the exciton diffusion as well. Therefore, these two parts of the exciton energy spectrum are of primary importance for the low-temperature exciton transport.

A. Analyzing the Low-Energy Structure. Here, we recall briefly the concept of the local (hidden) energy structure of localized 1D excitons, ${ }^{17,31,32}$ which was proved to exist in the vicinity of the band bottom. ${ }^{19,34}$ According to this concept, the low-energy one-exciton eigenfunctions obtained for a fixed realization of disorder are localized at segments of typical size $N^{*}$ (localization length). Some of these localized states (about $30 \%$ ) can be grouped into local manifolds of two (or sometimes

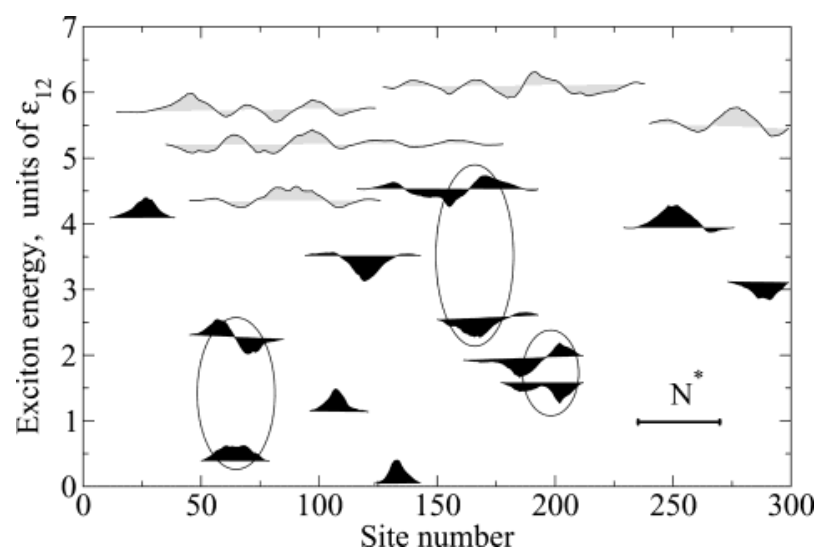

Figure 1. The energy structure of the exciton states in the vicinity of the bottom of the exciton band. The states are obtained by diagonalization of the Hamiltonian (eq 1) for a linear chain of 300 molecules and the disorder magnitude $\Delta=0.1 J$. The baseline of each state represents its energy in units of the spacing in the local energy structure, $\epsilon_{12}$. The origin of the exciton energy is set to the lowest energy for the realization. The wave functions are in arbitrary units. The typical localization length $N^{*}$ is given by the bar in the lower right corner.

more) states that are localized at the same $N^{*}$ molecule segment (see the states filled with black color and joined by ellipses in Figure 1). It turns out that the structure of the exciton states in each local manifold is very similar to the structure of the lower states of a homogeneous (nondisordered) linear chain of length $N^{*}$. In particular, the lowest state in a manifold has a wave function without nodes within its localization segment. Such a state can be interpreted as the local ground state of the segment (italic is used to distinguish this state from the true ground state, that is, the state with the lowest energy in each realization). A local ground state carries large oscillator strength, approximately $N^{*}$ times larger than that of a monomer, so the typical spontaneous emission rate is $\gamma^{*}=\gamma N^{*}$. The scaling law of the localization length is ${ }^{34}$

$$
N^{*}=8.7\left(\frac{\Delta}{J}\right)^{-0.67}
$$

The energy distribution of the local ground states, calculated as described in ref 34, and the total DOS are presented in Figure 2. This figure shows that almost all local ground states belong to the tail of the DOS, as has been mentioned in the Introduction.

The second state in a manifold has a node within the localization segment (see Figure 1) and looks like the first local excited state of the segment. Its oscillator strength is typically an order of magnitude smaller than that of the local ground state. It is important to recall here that, contrary to the eigenstates from the same manifold, the lower states localized at different segments overlap weakly (see all states filled with black color in Figure 1). The energies of the local ground states are distributed within the interval $\sqrt{2} \sigma_{11}$ ( $\sigma_{11}$ being the typical spacing between local ground states). This interval is larger than the typical energy spacing $\epsilon_{12}$ between the levels in a local manifold: ${ }^{34}$

$$
\begin{aligned}
& \sigma_{11}=0.7 J\left(\frac{\Delta}{J}\right)^{1.33} \\
& \epsilon_{12}=0.4 J\left(\frac{\Delta}{J}\right)^{1.36}
\end{aligned}
$$

For this reason, the local energy structure cannot be seen either in the DOS (see Figure 2) or in the linear absorption spectra 

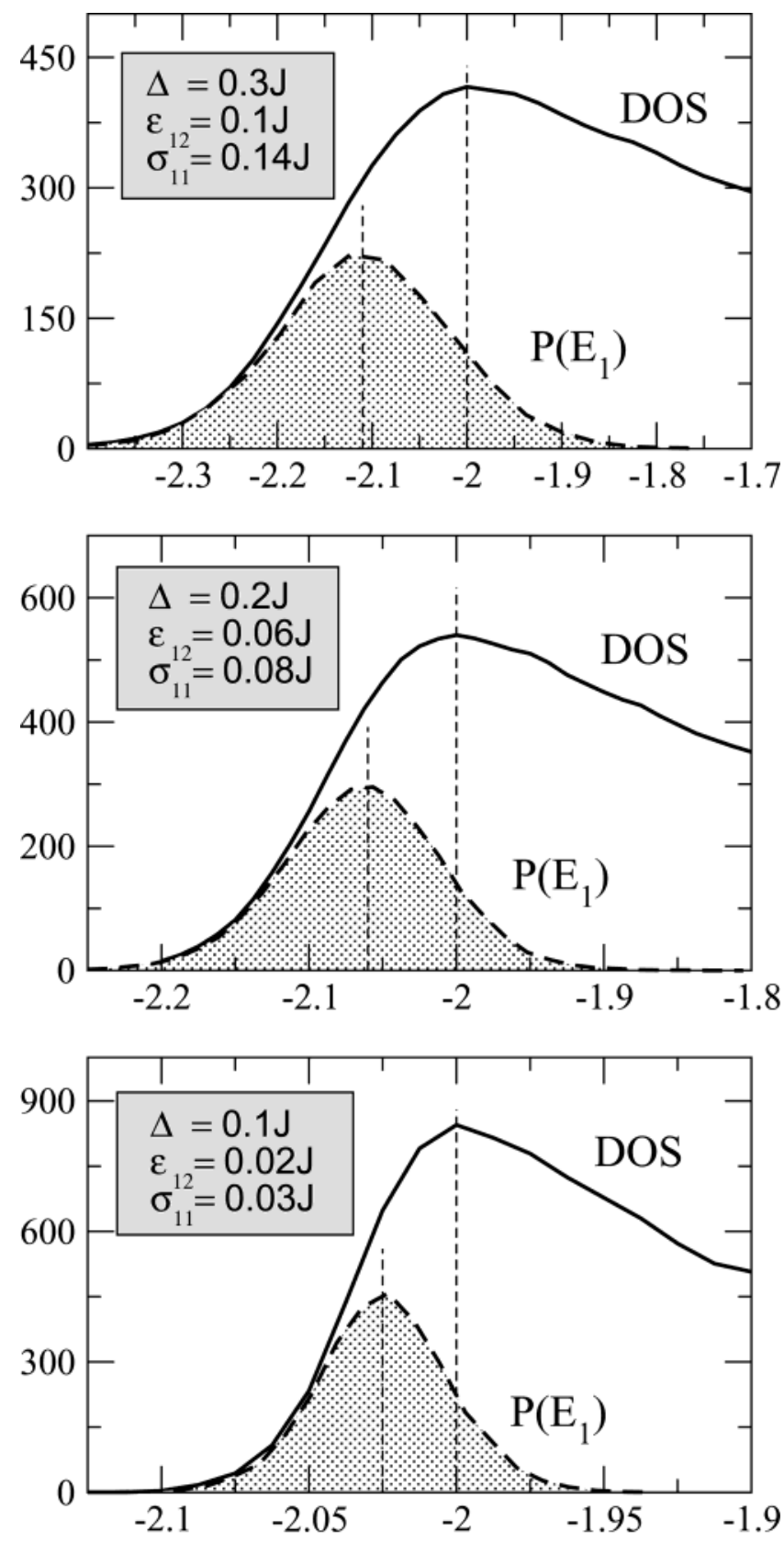

\section{Energy, units of $\mathrm{J}$}

Figure 2. The total DOS (-) and the DOS of the local ground states $P\left(E_{1}\right)(---)$ for different magnitudes of disorder $\Delta$. The DOS is normalized to $N ; P\left(E_{1}\right)$ is normalized to $N / N^{*}$. The vertical lines indicate positions of the curve maxima. For all considered magnitudes of disorder, the maximum of the local DOS is shifted with respect to the maximum of the total DOS by about the mean spacing in the local energy structure, $\epsilon_{12}$.

(see, for instance, ref 10). However, this structure determines the nonlinear optical response of the aggregate. ${ }^{35-37}$

Higher states are more extended than the local states because the localization length increases with energy (see the states filled with gray color in Figure 1). Therefore, the higher states cannot be included into any particular local manifold: their wave functions cover more than one $N^{*}$ molecule segment. Nevertheless, because all of these states are close to the maximum of the DOS (see Figure 2), the typical energy spacing between the higher states and the covered local states is of the order of $\epsilon_{12}$. Thus, the energy $\epsilon_{12}$ is expected to be the characteristic energy of the exciton diffusion.
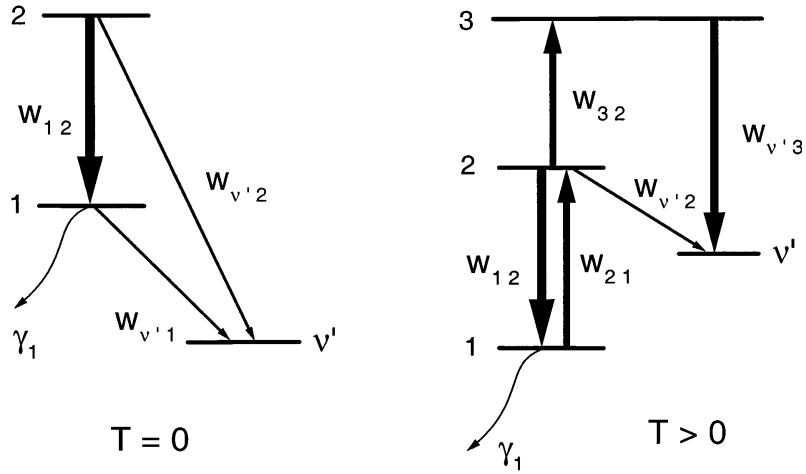

Figure 3. Schematic view of exciton hopping at zero and nonzero temperatures. The indices 1 and 2 label the local ground and the first local excited states of the same segment, respectively. The $v^{\prime}$ state is localized at an adjacent segment. The index 3 labels a higher state, which extends over two adjacent segments. Hops are shown by straight arrows; the arrow thickness represents magnitude of the coresponding hopping rate. Thin wavy arrows show spontaneous emission.

It is clear from the above arguments that at temperatures $T$ $\ll \epsilon_{12}$, it is the states from the local manifolds that determine the exciton diffusion. Two types of hopping over these states can be distinguished: intrasegment hopping and intersegment one, involving the states of the same local manifold and those of different manifolds, respectively. Because the states from different local manifolds overlap weakly, only intersegment hops to adjacent segments are of importance. The disorder scaling of the overlap integrals, $I_{\mu \nu}=\sum_{n} \varphi_{\mu n}{ }^{2} \varphi_{\nu n}{ }^{2}$ for the local states of the same and adjacent segments was obtained in ref 19:

$$
\begin{gathered}
I_{12}=0.14\left(\frac{\Delta}{J}\right)^{0.70} \\
I_{v^{\prime} 1} \approx I_{v^{\prime} 2}=0.0025\left(\frac{\Delta}{J}\right)^{0.75}
\end{gathered}
$$

Hereafter, the indices 1 and 2 label the local states of the same segment, while those with primes label the local states of an adjacent segment. As follows from eq 13, the intrasegment overlap integral is typically 2 orders of magnitude larger than the intersegment one. Note that both overlap integrals scale approximately proportional to the inverted $N^{*}$ [see eqs 11 and 13]. This proportionality holds for two exponential functions extended over the length $N^{*}$ and separated by the distance of the same order of magnitude.

The intrasegment hops do not result in the spatial displacement of excitons. Only the intersegment hopping gives rise to the spatial motion. Nevertheless, we show below that both types of hops are important for understanding the features of the lowtemperature exciton transport.

The overlap integrals between the local states of a segment and the higher states that are extended over this segment and a few adjacent ones (see the states filled with gray color in Figure 1) are on the order of $I_{12}$. This fact implies that even at $T<\epsilon_{12}$ the indirect hops via these higher states can be more efficient than the direct intersegment hops over the states of local manifolds (see below). Our calculations support this assumption.

B. Hopping at Zero Temperature. At zero temperature, an exciton can hop only down to lower states. Let us assume that it is in the local excited state 2. Then it can either hop to the local ground state 1 of the same segment or to a lower state $v^{\prime}$ localized at an adjacent segment (see Figure 3, $T=0$ ). Because the intrasegment hopping is faster than the intersegment one, first, the exciton hops down to the local ground state 1 with 
the typical energy loss $\epsilon_{12}\left(\epsilon_{12}\right.$ being the mean energy spacing in the local energy structure). From the local ground state 1, the exciton can hop only to a state $v^{\prime}$ of an adjacent segment, provided that $\epsilon_{v^{\prime}}<\epsilon_{1}$ and the spontaneous emission rate of the local ground state $\gamma_{1}$ is small compared to the intrasegment hopping rate, $\left.W_{v^{\prime} 1}\right|_{T=0}$. Hereafter, such a relationship between these rates is referred to as the limit of fast diffusion; only this limit is considered in this work. The typical energy loss during such sideways hop is on the order of the average spacing between local ground states, $\sigma_{11}\left(\sigma_{11}\right.$ is on the order of the J-bandwidth). Thus, already after one such sideways hop, the exciton resides in a state in the tail of the DOS (see Figure 2). Therefore, the number of states with even lower energies decreases dramatically, which results in a strong increase of the typical distance to those states and decrease of the probability to hop further sideways. Then the exciton either relaxes to a lower state of the same segment (if there is one) or decays spontaneously, that is, this type of the spatioenergetic diffusion (toward lower energies) stops very quickly. Note that this diffusion would manifest itself in the red shift of the exciton emission spectrum relative to the absorption spectrum. The experimental data shows that such red shift is either absent ${ }^{6,7}$ or is smaller than the J-bandwidth. ${ }^{23,38}$ These experimental facts indicate unambiguously that at low temperatures, $T \ll \sigma_{11}$, excitons make few hops before they decay because of the spontaneous emission, as was argued in refs 39-41. Consequently, the zero-temperature exciton quenching is expected to be weak provided the concentration of quenchers is low, the case that we are interested in.

C. Hopping at Nonzero Temperatures. At nonzero but low temperatures $\left(0<T \lesssim \epsilon_{12}\right)$, an exciton can also hop up in energy. Consider an exciton in one of the lower states in the tail of the DOS, for example, in the local ground state 1 (see Figure 3, $T>0$ ). For the reasons discussed above, first, the exciton hops up to the first local excited state 2 of the same segment, provided that the hopping rate for the considered temperature is larger than the spontaneous emission rate $\gamma_{1}$ of the initial state 1 . During this process, the exciton typically gains the energy $\epsilon_{12}$. Because $\epsilon_{12}$ is on the order of $\sigma_{11},{ }^{34}$ already after the first hop up the exciton leaves the tail of the DOS (see Figure 2), and hence, it is likely to have a lower state $v^{\prime}$ localized at an adjacent segment. A hop down to this state with loss in energy is favorable and results in the spatial displacement of the exciton, that is, in the exciton diffusion. We stress that although only sideways hops result in the spatial displacement of the exciton, it is the initial hop up from the local ground state 1 to the local excited state 2 that triggers the diffusion.

Another way for the exciton to hop sideways to the state $v^{\prime}$ is via the higher state 3 that overlaps well with both states 2 and $v^{\prime}$ (see Figure 3,T>0). As it has been mentioned, such hops compete with the sideways hops over the local states; although the hop up to the state 3 is thermally unfavorable, the overlap integral for this hop, $I_{31}$, is large compared to that for an intersegment hop, $I_{v^{\prime} 1}$. We show later that this channel of the diffusion becomes dominant even at relatively low temperature.

\section{Temperature Dependence of the Quenching Rate}

In this section, we discuss the results of numerical calculation of the quenching rate $W_{\mathrm{q}}$. We consider the initial condition in which the leftmost local ground state is excited while a single trap is located in the center of the localization segment of the rightmost local ground state. In this case, the exciton quenching is most affected by the diffusion because the created exciton has to travel over almost the whole chain to be quenched. Thus, the exciton quenching at low concentration of traps can be studied. The quenching rate was calculated as described in section 2 for the parameter set corresponding to the limit of fast diffusion and fast quenching (the latter limit is defined subsequently).

A. Numerical results. As it was already mentioned in section 3 , in the limit of fast diffusion the intersegment down-hopping rate is large compared to the typical spontaneous emission rate of a local ground state:

$$
\left.W_{v^{\prime} 1}\right|_{T=0} \approx W_{0} \frac{\sigma_{11}}{J} I_{v^{\prime} 1} \gg \gamma N^{*}
$$

If a quencher is located within the localization segment of a local state, then the typical quenching rate for this state is $\Gamma^{*}$ $=\Gamma / N^{*}$ [see eq 4]. Because we are interested in the limit of fast quenching, this rate should be taken to be larger than the typical intrasegment down-hopping rate:

$$
\Gamma /\left.N^{*} \gg W_{12}\right|_{T=0} \approx W_{0} \frac{\epsilon_{12}}{J} I_{12}
$$

This ensures that once an exciton hops to a local state of the segment with the trap, it is quenched almost instantly.

The inequalities of eqs 14 and 15 yield the relationship between the rate equation parameters in the limit of fast diffusion and quenching:

$$
\begin{gathered}
W_{0} \frac{\sigma_{11}}{J} \frac{I_{1^{\prime} 2}}{N^{*}} \gg \gamma \\
W_{0} \frac{\epsilon_{12}}{J} I_{12} N^{*} \ll \Gamma
\end{gathered}
$$

On the basis of the scaling laws, eqs 12 and 13, the parameter set was chosen so that inequalities in eq 16 hold. More specifically, for each magnitude of the disorder, $\left.W_{1^{\prime} 1}\right|_{T=0}=10 \gamma^{*}$ and $\Gamma^{*}=\left.10 W_{12}\right|_{T=0}$ where $\gamma=5 \times 10^{-8}$ and $\Gamma=0.2$ for $\Delta$ $=0.1 J, \gamma=4 \times 10^{-7}$ and $\Gamma=0.5$ for $\Delta=0.2 J$, and $\gamma=1 \times$ $10^{-6}$ and $\Gamma=0.9$ for $\Delta=0.3 J$. In all calculations, we set $W_{0}$ $=1$. Calculations were performed for $N=1000$ and averaged over 100 realizations of the disorder (increasing the number of realizations of disorder leads to negligible changes of the results).

Figure 4 shows the temperature dependence of the quenching rate $W_{\mathrm{q}}$ for different magnitudes of the disorder $\Delta$. In each plot, the quenching rate is given in units of the typical exciton radiative rate $\gamma^{*}=\gamma N^{*}$. The temperature is given in units of the mean energy spacing in the local energy structure $\epsilon_{12}$. Note that both $N^{*}$ and $\epsilon_{12}$ depend on $\Delta$ as described by eqs 11 and $12 \mathrm{~b}$. Figure 4 demonstrates very clearly that for all considered values of $\Delta$ at temperatures lower than $\epsilon_{12}$ the quenching rate is vanishing. This indicates that the diffusion at these temperatures is not fast enough for the exciton to reach the quencher during its (spontaneous) lifetime: it emits a photon before it is trapped. In contrast, just after the temperature exceeds approximately $\epsilon_{12}$, the quenching becomes noticeable: the exciton partly diffuses to the trap where it decays mostly because of quenching. Specifically, temperatures on the order of $2 \epsilon_{12}$ are required for the quenching to become as effective as the spontaneous emission: $W_{\mathrm{q}} \approx \gamma^{*}=\gamma N^{*}$.

B. Discussion. To understand which states contribute most to the quenching process, it is useful to estimate the effective sideways hopping rate $W$ that is required to reach the quenching 


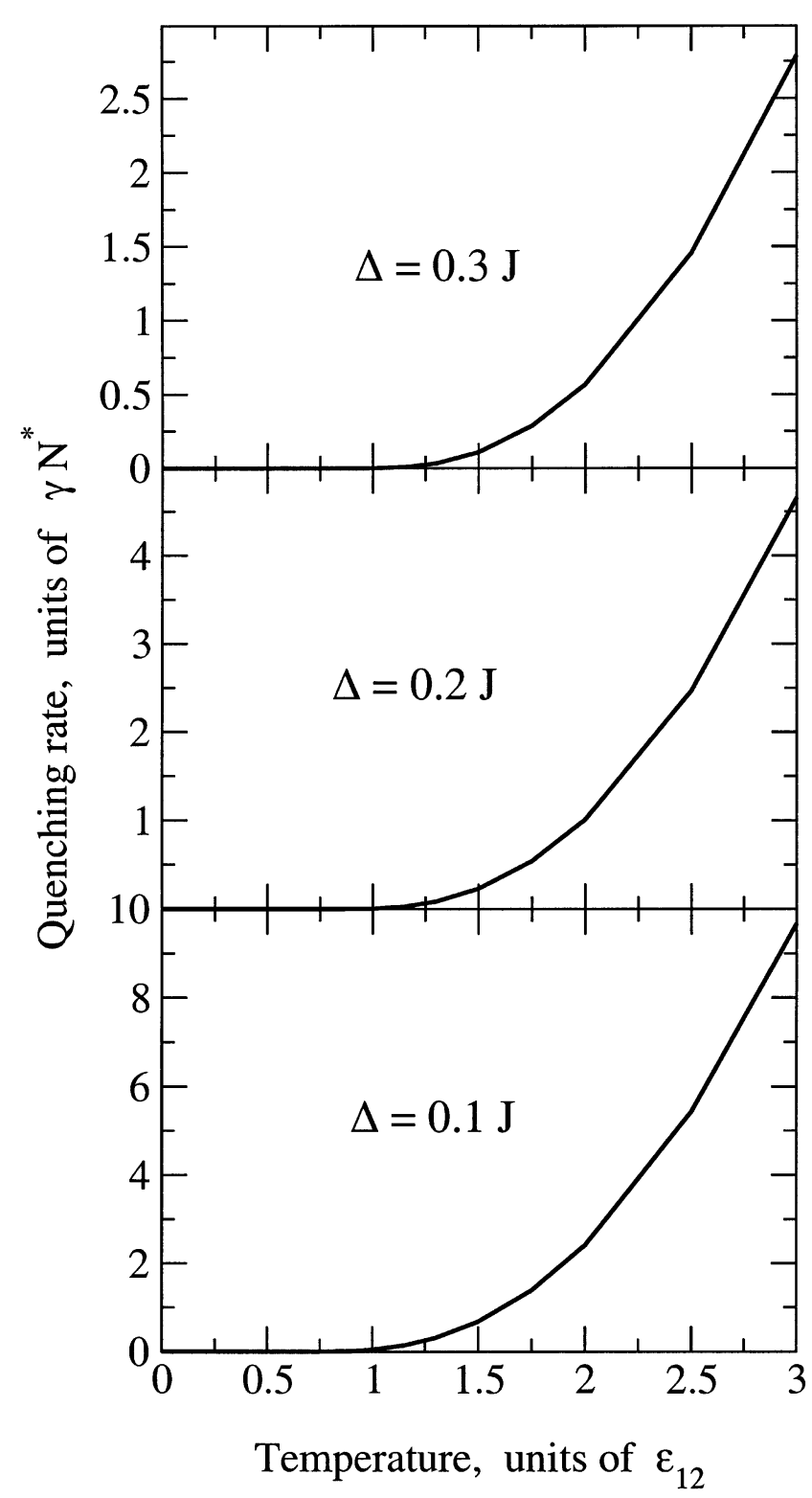

Figure 4. Temperature dependence of the quenching rate $W_{\mathrm{q}}$ calculated for a linear chain of the length $N=1000$ and different magnitudes of the disorder $\Delta$. The averaging is performed over 100 disorder realizations. For each realization of the disorder, the leftmost local ground state is excited, while the only trap is located in the center of the localization segment of the rightmost local ground state.

level $W_{\mathrm{q}} \approx \gamma^{*}$. To do this, consider the sequence of localization segments as an effective chain of "sites", the typical number of which is equal to the number of segments, $N_{\mathrm{s}}=N / N^{*}$; the mean spacing between these "sites" is $N^{*}$. The exciton diffusion coefficient is then estimated as $D \approx W N^{* 2}$ (the lattice constant is set to unity). The quenching is as effective as the spontaneous decay if the exciton reaches the trap (located on the opposite side of the chain) during its lifetime $\gamma^{*-1}$, that is, it has to diffuse over the distance $N$ during this time. Equating the diffusion length $\sqrt{D / \gamma^{*}}$ to $N$, we obtain the estimate for the required diffusion rate $W$ :

$$
W \approx \gamma^{*}\left(N / N^{*}\right)^{2}
$$

The localization length $N^{*}$ is equal to 38,25 , and 18 for $\Delta=$ $0.1,0.2$ and 0.3 , respectively. Thus, the corresponding diffusion rates $W$ are estimated as $625 \gamma^{*}, 1600 \gamma^{*}$, and $2500 \gamma^{*}$ (for $N=$ 1000). These values are about 2 orders of magnitude larger than

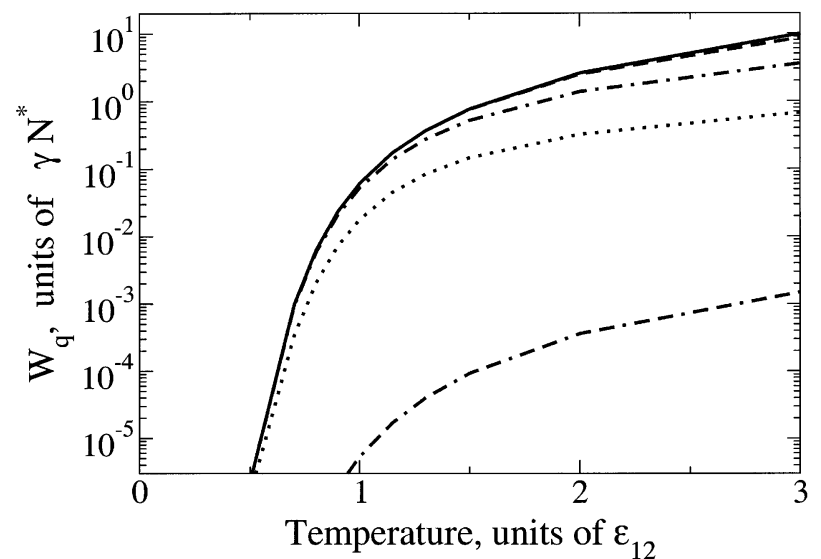

Figure 5. Temperature dependence of the quenching rate $W_{\mathrm{q}}$ calculated for $\Delta=0.1 J\left(N^{*} \approx 40\right)$ and different numbers of states considered in eq 9: (-) all $N=1000$ states; $(---) 6 N / N^{*}=150$ states; $(-\cdot-)$ $4 N / N^{*}=100$ states; $(\cdots) 3 N / N^{*}=75$ states; $(--\cdot) 2 N / N^{*}=50$ states. The averaging is performed over 100 disorder realizations. For each realization of the disorder, the leftmost local ground state is excited, while the only trap is located in the center of the localization segment of the rightmost local ground state.

the rates of sideways hops over the local states, taken to be $10 \gamma^{*}$ in all calculations. This indicates that when the quenching rate becomes comparable to the spontaneous emission rate, the exciton does not hop directly between the local states of adjacent segments (with the typical rate $W_{v^{\prime} 1} \approx 10 \gamma^{*}$ ). It rather hops via the higher states that extend over more than one $N^{*}$ molecule segments (see the discussion in section 3 ). The hopping rate via such states for $T \approx 2 \epsilon_{12}$ is on the order of $W_{12}$, which is about 2 orders of magnitude larger than $W_{v^{\prime} 1}$ [see eq 3 and the scaling laws (eq 13)].

To prove the above finding, we performed calculations of the quenching rate $W_{\mathrm{q}}$ varying the number of states considered in eq 10. More specifically, we considered all states up to some (variable) cutoff state. Figure 5 shows the results of such study performed for $\Delta=0.1 J$. As it can be seen from the figure, $W_{\mathrm{q}}$ depends drastically on the number of states participating in the quenching process. In the region where $W_{\mathrm{q}}>\gamma^{*}$, approximately $6 N / N^{*}$ states are required to reach the true value of the quenching rate that is calculated for all states (compare dashed and solid lines). Thus, at temperatures $T \gtrsim \epsilon_{12}$, the higher states provide the dominant contribution to the exciton quenching process.

Figure 6 shows the regions of the DOS that correspond to different numbers of states that were used for calculation of the data presented in Figure 5. The higher states lie just after the local ones, close to the maximum of the DOS (see Figure $6)$. Therefore, the typical energy spacing between the local and higher states is about $\epsilon_{12}$. Because the higher states extend over several, but not very many, $N^{*}$ molecule segments (see Figure $1)$, the overlap integral between these states and the covered local states is large. These two factors ensure high hopping rate from the lower local to the higher states. Another important point is that higher states are well-overlapped and more extended, so hops between them are typically faster and longer than those between the local ones. Also, the higher states have small oscillator strength, so as long as an exciton remains in these states, it does not decay radiatively. The above qualitative arguments explain the dominant contribution of the higher states into the exciton diffusion and quenching within the temperature range $T \gtrsim \epsilon_{12}$.

It is also seen from Figure 5 that the difference between the true value of the quenching rate $W_{\mathrm{q}}$ and that calculated for a 


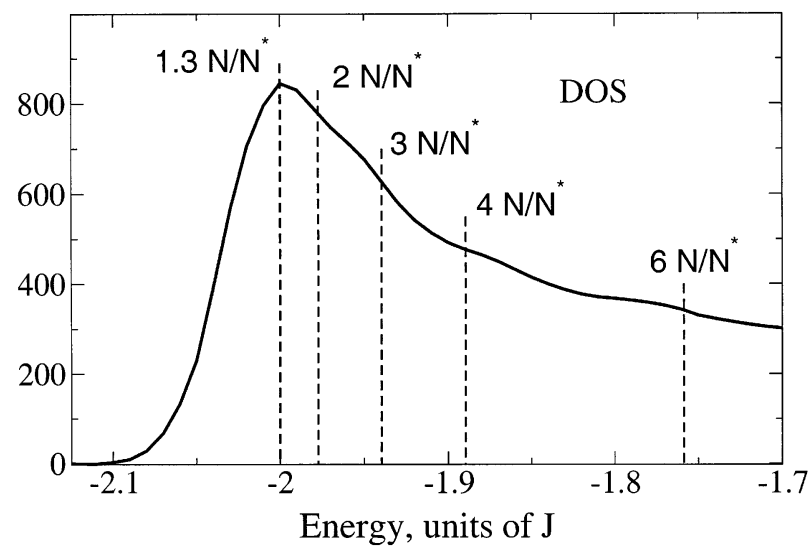

Figure 6. The total DOS calculated for $N=1000$ and $\Delta=0.1 J\left(N^{*}\right.$ $\approx 40$ ). The DOS is normalized to $N$. The vertical lines show the maximum energies corresponding to different numbers of states considered in the rate equation that was used to calculate the dependencies plotted in Figure 5 (in the sense that all states lower than the specified energy are considered). Note that the tail of the DOS is formed by $1.3 N / N^{*}$ states, namely, by the states of the local manifolds $\left(N / N^{*}\right.$ local ground states plus $0.3 N / N^{*}$ of the local excited states; recall that about $30 \%$ of the local ground states form the doublets).

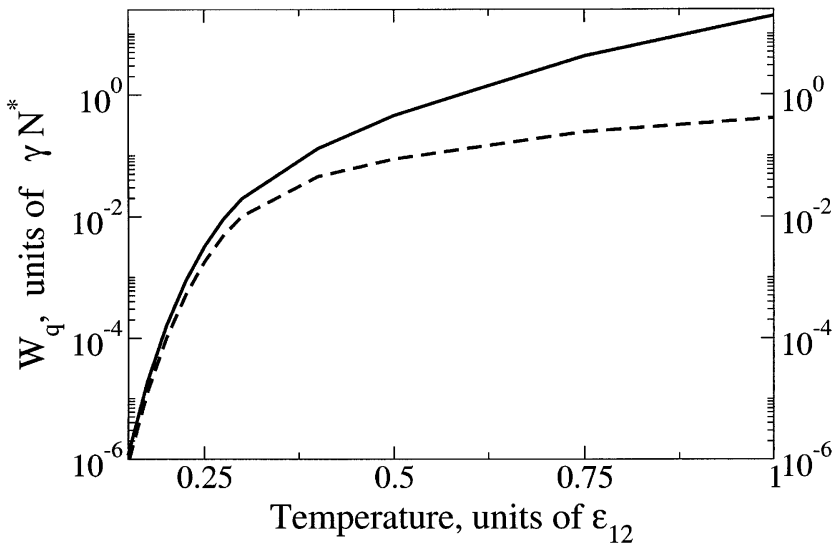

Figure 7. Temperature dependence of the quenching rate $W_{\mathrm{q}}$ calculated for $\Delta=0.2 J\left(N^{*} \approx 25\right)$ : $(-)$ all $N=250$ states; $(---) 2 N / N^{*}=$ 20 states.

restricted number of states decreases at lower temperatures. Figure 7 demonstrates the temperature dependence of $W_{\mathrm{q}}$ obtained for $\Delta=0.2 J\left(N^{*} \approx 25\right)$ and temperatures $T<\epsilon_{12}$. The solid line presents the dependence calculated for all $N=$ 250 states considered in the rate equation and the dashed line that for $2 N / N^{*}=20$ states. These $2 N / N^{*}$ states include all of the states of the local manifolds $1.3 N / N^{*}$ and $0.7 N / N^{*}$ higher ones.

The parameters of the rate equation were set as follows: $\Gamma^{*}$ $=\left.10 W_{12}\right|_{T=0}$ and $\left.W_{1^{\prime} 1}\right|_{T=0}=100 \gamma^{*}$. The chain length $N=250$ is chosen so that the effective hopping rate $W \approx \gamma^{*}\left(N / N^{*}\right)^{2}$ estimated as discussed above (see eq 17) is equal to the rate of the direct hopping to an adjacent segment, $\left.W_{1^{\prime} 1}\right|_{T=0}$. This yields the equation $\left(N / N^{*}\right)^{2}=100$ for the chain length. This condition ensures that the diffusion over the lower states only can provide the quenching rate $W_{\mathrm{q}} \approx \gamma^{*}$ at $T \approx \epsilon_{12}$. Indeed, $W_{\mathrm{q}} \approx 0.5 \gamma^{*}$ at this temperature for $2 N / N^{*}$ states. The most important point demonstrated by Figure 7 is that below the temperature $T_{1} \approx$ $0.25 \epsilon_{12}$ the two curves deviate slightly, which means that the contribution of the higher states into the diffusion becomes negligible: the exciton hops mostly over the DOS tail states. In contrast, above the temperature $T_{1}$, the higher states provide the dominant contribution to the diffusion and quenching. Note also that the value of the quenching rate at the critical temperature $T_{1}$ is typically very small, so the experimental observation of this "regime change" is a challenging task.

The critical temperature $T_{1}$ at which the higher states come into play can be estimated by equating the typical rate of the direct sideways hopping from a local state 2 to an adjacent local state 1 ' to the "vertical" hopping rate from the local state 2 to a higher state 3 (see Figure 3, $T>0$ ): $W_{1^{\prime} 2}=\left.W_{32} \approx W_{12}\right|_{T=0}$ $\exp \left(\epsilon_{12} / T_{1}\right)$. This equation yields the temperature $T_{1}$ :

$$
T_{1}=\frac{1}{\ln \left(I_{12} / I_{1^{\prime} 2}\right)} \epsilon_{12} \approx 0.25 \epsilon_{12}
$$

We stress that the numerical factor $1 / \ln \left(I_{12} / I_{1^{\prime} 2}\right) \approx 0.25$ is almost independent of the disorder because the disorder scalings of the overlap integrals are almost the same (see eq 13). So, the estimate $T_{1} \approx 0.25 \epsilon_{12}$ is universal for a wide range of the disorder degree.

\section{Summary and Concluding Remarks}

In this paper, we study theoretically the peculiarities of the low-temperature diffusion of the 1D Frenkel excitons localized by a moderate diagonal disorder. The exciton motion over localized states is considered as incoherent hopping. The diffusion is probed by the exciton quenching at a point trap. We consider a single trap located at one end of the aggregate, while the exciton is created initially at the other end. In this case, the exciton has to travel over almost the whole chain to be quenched. Under these conditions, the quenching rate carries direct information about the diffusion length that the exciton travels during its lifetime. The exciton quenching is described by the rate equation with the quenching rate being proportional to the probability of finding the exciton at the trap site.

Both our qualitative arguments and numerical simulations show that there exist two regimes of the exciton diffusion. At lower temperatures, those smaller than $T_{1} \approx 0.25$ (J-bandwidth), the exciton diffuses mostly over weakly overlapped DOS tail states, which determine the optical response and form the J-band. This regime of diffusion is very slow; the exciton cannot diffuse over large distance during its lifetime at these temperatures.

At higher temperatures, the higher states come into play. The diffusion begins to build up because of the two-step hops via higher states. This accelerates the exciton diffusion drastically, so an exciton can diffuse over large distances during its lifetime. The higher states begin to contribute dominantly to the diffusion at temperatures higher than about $T_{1}$. However, the diffusion becomes really fast (in the sense that the quenching rate becomes comparable to the spontaneous emission rate of the aggregate) only at the temperatures on the order of the J-bandwidth.

In ref 23 , the anomalously fast low-temperature diffusion of Frenkel excitons in J-aggregates of THIATS was reported. The unit cell in these aggregates contains two THIATS molecules. Because of this fact, the absorption spectra of THIATS aggregates reveal two bands, so-called H-band and J-band. ${ }^{42}$ The former, intensive and widely broadened (the width being about $1000 \mathrm{~cm}^{-1}$ ), results from the optical transition from the ground state of the aggregate to the top of the exciton band. The latter, much less intensive and narrower (the width being $82 \mathrm{~cm}^{-1}$ ), is due to the optical transition from the ground state to the bottom of the exciton band. Contrary to the H-band, the $\mathrm{J}$-band is visible in exciton fluorescence spectra.

The authors of ref 23 studied experimentally the excitonexciton annihilation and found that this effect is pronounced even at $T=5 \mathrm{~K}\left(3.5 \mathrm{~cm}^{-1}\right)$, while the width of J-band of THIATS J-aggregates is $82 \mathrm{~cm}^{-1}$. To explain the experimental 
data, the authors assumed that an exciton travels over about $10^{4}$ dye molecules during its lifetime to meet another exciton and annihilate. They found also that the activation energy of the exciton diffusion was $15 \mathrm{~K}\left(10.5 \mathrm{~cm}^{-1}\right)$ and interpreted this energy as the typical energy spacing between the states of adjacent localization segments.

The fact that the exciton-exciton annihilation is very sensitive to temperature indicates that this process starts after the exciton intraband relaxation to the states forming the J-band. Therefore, the annihilation process involves only the J-band states, and the difference in band structure between THIATS aggregates and J-aggregates is probably unimportant. Furthermore, the exciton-exciton annihilation can be treated similarly to the exciton quenching: one of the two excitons can be considered as an immobile trap for the other, while the other diffuses twice as fast. Thus, our model is applicable to analyzing the excitonexciton annihilation in THIATS aggregates. As reported in ref 23, the fluorescence spectrum of THIATS J-aggregates is narrowed by approximately $26 \mathrm{~cm}^{-1}$ and red-shifted by $23 \mathrm{~cm}^{-1}$ as compared to the absorption spectrum. These results indicate unambiguously that excitons make sideways hops during their lifetime, that is, the rate of sideways hops over local states is larger than the exciton spontaneous emission rate. Thus, the conditions for the exciton diffusion in THIATS J-aggregates are similar to those studied in the present paper (the limit of fast diffusion).

Through discussion of the above-mentioned experimental data and its interpretation presented in ref 23 , the following points can be made. First, the typical energy spacing between the states of the adjacent segments is on the order of the J-bandwidth, ${ }^{19}$ that is 82 and not $10.5 \mathrm{~cm}^{-1}$. The latter value is closer to 0.25 $\times 82 \mathrm{~cm}^{-1}$, that is, this temperature could be related to the temperature $T_{1}$, the activation energy of the faster exciton diffusion regime. Above this temperature, an exciton diffuses mostly over the higher states and not over the DOS tail states, as it was suggested in ref 23. Another point, and a more important one, is of the quantitative nature: the typical size of the localization segment in THIATS J-aggregates is $N^{*}=30 .{ }^{42}$ In the model that we are using, this corresponds to the disorder magnitude $\Delta \approx 0.2 \mathrm{~J}$. Our numerical data obtained for a chain of $N=1000$ molecules demonstrates that for this value of the disorder the exciton quenching is vanishingly small for the temperatures $T \approx(10.5 / 82) \epsilon_{12}$ (we remind that $\epsilon_{12}$ is on the order of the J-bandwidth). In other words, the exciton created in the leftmost local ground state cannot diffuse over the whole chain of 1000 monomers during its lifetime. However, it can do so at the temperatures on the order of $T \approx \mathrm{e}_{12} \approx 82 \mathrm{~cm}^{-1}$. Thus, understanding the fast low-temperature diffusion in the aggregates of THIATS dye molecules, observed in ref 23, still remains an open question.

Acknowledgment. This work was supported by the DGIMCyT (Project MAT2000-0734). A.V.M. and F.D.A. acknowledge support from CAM (Project 07N/0075/2001). V.A.M. acknowledges support from MECyD (Project SAB2000-0103) and through a NATO Fellowship.

\section{References and Notes}

(1) Jelley, E. E. Nature (London) 1936, 38, 1009.

(2) Scheibe, G. Angew. Chem. 1936, 49, 563.
(3) Frenkel, J. Phys. Rev. 1931, 37, 17 1971

(4) Davydov, A. S. Theory of Molecular Excitons; Plenum: New York,

(5) Agranovich, V. M.; Galanin, M. D. In Electronic Excitation Energy Transfer in Condensed Matter; Agranovich, V. M., Maradudin, A. A., Eds. North-Holland: Amsterdam, 1982.

(6) de Boer, S.; Wiersma, D. A. Chem. Phys. Lett. 1990, 165, 45

(7) Fidder, H.; Knoester, J.; Wiersma, D. A. Chem. Phys. Lett. 1990, 171,529 .

(8) Fidder, H.; Terpstra, J.; Wiersma, D. A. J. Chem. Phys. 1991, 94, 6895.

(9) Fidder, H.; Ph.D. Thesis, University of Groningen, Groningen, Netherlands, 1993.

(10) Fidder, H.; Knoester, J.; Wiersma, D. A. J. Chem. Phys. 1991, 95,7880 .

(11) Spano, F. C.; Knoester, J. In Advances in Magnetic and Optical Resonance; Warren, W. S., Ed.; Academic: New York, 1994; Vol. 18, p 117.

(12) Contributions to Adv. Mater. 1995, 7.

(13) J-aggregates; Kobayashi, T., Ed.; World Scientific: Singapore, 1996.

(14) Knapp, E. W. Chem. Phys. 1984, 85, 73.

(15) Knoester, J. J. Chem. Phys. 1993, 99, 8466; J. Lumin. 1994, 58, 107.

(16) Durrant, J. R.; Knoester, J.; Wiersma, D. A. Chem. Phys. Lett. 1994, 222, 450

(17) Malyshev, V. A.; Rodríguez, A.; Domínguez-Adame, F. Phys. Rev. $B$ 1999, 60, 14140 .

(18) Malyshev, V. A.; Domínguez-Adame, F. Chem. Phys. Lett. 1999, 313,255 .

(19) Malyshev, A. V.; Malyshev, V. A. J. Lumin 2001, 369, 94-95

(20) Shklovskii, B. I.; Efros, A. L. Electronic Properties of Doped Semiconductors; Springer-Verlag: Berlin, 1984.

(21) Basiev, T. T.; Malyshev, V. A.; Przhevuskii, A. K. In Spectroscopy of Solids Containing Rare-Earth Ions; Kaplyanskii, A. A., MacFarlane, R. M., Eds.; North-Holland: Amsterdam, 1987; p 275.

(22) Malyshev, V. A. Fiz. Tv. Tela 1992, 34, 1109 [Transl. Phys. Solid State 1992, 34, 590].

(23) Scheblykin, I. G.; Sliusarenko, O. Yu.; Lepnev, L. S.; Vitukhnovsky, A. G.; Van der Auweraer, M. J. Phys. Chem. B 2000, 104, 10949 2001, 105, 4636 .

(24) Leegwater, J. A.; Durrant, J. R.; Klug, D. R. J. Phys. Chem. B 1997, 101, 7205.

(25) Bednarz, M.; Malyshev, V. A.; Knoester, J. J. Chem. Phys. 2002, 117,6200

(26) Dean, P. Rev. Mod. Phys. 1972, 44, 127.

(27) Ovsyankin, V. V. In Spectroscopy of Solids Containing Rare-Earth Ions; Kaplyanskii, A. A., MacFarlane, R. M., Eds.; North-Holland: Amsterdam, 1987; p 343.

(28) Shimizu, M.; Suto, S.; Goto, T. J. Chem. Phys. 2001, 114, 2775.

(29) Bednarz, M.; Malyshev, V. A.; Lemaistre, J. P.; Knoester, J. J. Lumin. 2001, 271, 94-95.

(30) Spano, F. C.; Kuklinsky, J. R.; Mukamel, S. Phys. Rev. Lett. 1990 65, 211; J. Chem. Phys. 1991, 94, 7534.

(31) Malyshev, V. A. Opt. Spektrosk. 1991, 71, 873 [Transl. Opt. Spectrosc. 1991, 71, 505]; J. Lumin. 1993, 55, 225.

(32) Malyshev, V.; Moreno, P. Phys. Rev. B 1995, 51, 14587

(33) Shimizu, M.; Suto, S.; Goto, T.; Watanabe, A.; Matsuda, M. Phys.

Rev. B 1998, 58, 5032 .

(34) Malyshev, A. V.; Malyshev, V. A. Phys. Rev. B 2001, 63, 195111.

(35) Minoshima, K.; Taiji, M.; Misawa, K.; Kobayashi, T. Chem. Phys. Lett. 1994, 218, 67.

(36) Knoester, J.; Spano, F. C. In J-aggregates; Kobayashi, T., Ed.; World Scientific: Singapore, 1996; p 111.

(37) Bakalis, L. D.; Knoester, J. J. Phys. Chem. B 1999, 103, 6620; J. Lumin. 2000, 66, 87-89.

(38) Kamalov, V. F.; Struganova, I. A.; Ioshihara, K. J. Phys. Chem. 1996, $100,8640$.

(39) Malyshev, V. A.; Glaeske, H.; Feller, K.-H. Chem. Phys. Lett. 1999, 305, 117

(40) Malyshev, V. A.; Kozlov, G. G.; Glaeske, H.; Feller, K.-H. Chem. Phys. 2000, 254, 31.

(41) Ryzhov, I. V. Kozlov, G. G.; Malyshev, V. A.; Knoester, J. J. Chem. Phys. 2001, 114, 5322.

(42) Scheblykin, I. G.; Bataiev, M. M.; Van der Auweraer, M. Vitukhnovsky, A. G. Chem. Phys. Lett. 2000, 316, 37. 